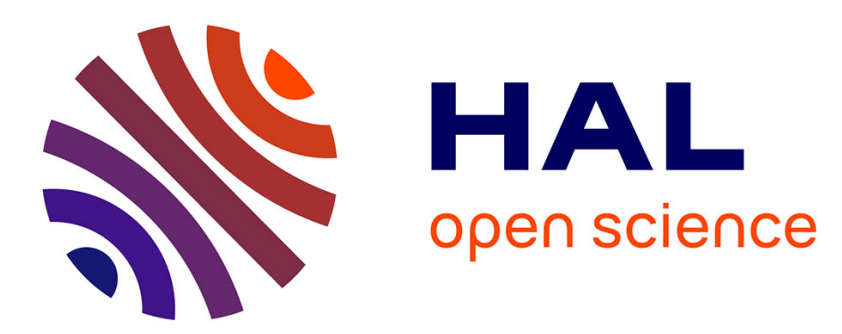

\title{
Sliding mode control synthesis of uncertain time-delay systems
}

Yuri Orlov, Wilfrid Perruquetti, Jean-Pierre Richard

\section{To cite this version:}

Yuri Orlov, Wilfrid Perruquetti, Jean-Pierre Richard. Sliding mode control synthesis of uncertain time-delay systems. Asian Journal of Control, 2003, 5 (4), pp.568-577. 10.1111/j.19346093.2003.tb00173.x . inria-00192158

\section{HAL Id: inria-00192158 https://hal.inria.fr/inria-00192158}

Submitted on 26 Nov 2007

HAL is a multi-disciplinary open access archive for the deposit and dissemination of scientific research documents, whether they are published or not. The documents may come from teaching and research institutions in France or abroad, or from public or private research centers.
L'archive ouverte pluridisciplinaire HAL, est destinée au dépôt et à la diffusion de documents scientifiques de niveau recherche, publiés ou non, émanant des établissements d'enseignement et de recherche français ou étrangers, des laboratoires publics ou privés. 


\title{
SLIDING MODE CONTROL SYNTHESIS OF UNCERTAIN TIME-DELAY SYSTEMS
}

\author{
Y. ORLOV ${ }^{*}$, W. Perruquetti ${ }^{\dagger}$, and J.P. Richard ${ }^{\dagger}$
}

December 5, 2002

\begin{abstract}
Sliding mode control synthesis is developed for a class of nonlinear time-delay systems. The synthesis is based on a new delay-dependent stability criterion. The controller constructed proves to be robust against sufficiently small delay variations and external disturbances. An admissible upperbound such that the corresponding closed-loop system remains globally asymptotically stable for each delay value less than this upperbound is derived. Performance issues of the controller are illustrated in a simulation study.
\end{abstract}

\section{Introduction}

The primary concern of the paper is robust control of uncertain time-delay systems within the framework of sliding mode control methods.

The sliding mode control paradigm implies the deliberate introduction of so-called sliding motions (i.e., the motions along a manifold where the control signal undergoes discontinuities) into the control system and it consists of two steps [21]. First, a manifold, such that if confined to this manifold the system has desired dynamic properties, is designed. Second, a discontinuous control law, which drives the system to this manifold in finite time, is synthesized.

The sliding mode control strategy is to construct a feedback that guarantees a Lyapunov function, selected for a nominal system, to remain negative on the trajectories of the perturbed system. The controller, thus constructed, asymptotically stabilizes the system and since the motion along the manifold proves to be uncorrupted by matched disturbances, the closed-loop system is

${ }^{*}$ CICESE Research Center, Electronics and Telecommunication Department, P.O.Box 434944 San Diego, CA 92143-4944 USA; FAX: +52(646)175-0554; Phone +52(646)175-0595; e-mail: yorlov@cicese.mx

${ }^{\dagger}$ LAIL, CNRS UPRESA 8021, Ecole Centrale de Lille, BP48, 59651 Villeneuve d'Ascq CEDEX-FRANCE; Fax: 33(3)20 3354 18; Phone: 33(3)20 335410 e-mail: wilfrid.perruquetti(jean-pierre.richard)@ec-lille.fr 
additionally guaranteed to have strong robustness properties against matched disturbances. Due to these advantages and simplicity of implementation, sliding mode controllers have widely been used in various applications [22].

Motivated by technological advances, the interest recently emerged in extending the sliding mode control approach to infinite-dimensional dynamic systems such as distributed parameter systems and time-delay systems. The earlier works $[17,25]$ on extensions of sliding mode control algorithms to infinitedimensional systems ran into a major difficulty, caused by the presence of an unbounded infinitesimal operator in the plant equation, and called for further theoretical investigations. Presently, the sliding mode control synthesis in the infinite-dimensional setting is well documented $[12,13,15,16]$. This synthesis retains robustness features, similar to those possessed by its counterpart in the finite-dimensional case, and being complementary to the $H_{\infty}$-design, it constitutes a more practical approach to infinite-dimensional systems than the ones of high computational complexity outlined in $[2,5,7,10]$.

The existing results $[6,8,9,18,23]$ on application of sliding mode control algorithms to time-delay systems, governed by functional differential equations (FDE), have corroborated their utility for this class of systems as well. However, these results, being inherited from the finite-dimensional treatment, contained no arguments supporting the well-posedness of FDE solutions to substantiate the stability analysis. Even the solution concept for discontinuous time-delay systems has not yet been addressed. This fundamental issue on the precise meaning of FDE with discontinuous right-hand side is resolved in the present paper, and substantial stability analysis and sliding mode control synthesis are subsequently developed for time-delay systems.

The outline of the paper is as follows. Plant description and formal problem statement appear in Section 2. After giving background materials on discontinuous time-delay systems in Section 3, Section 4 presents a new delay-dependent stability criterion for a class of nonlinear time-delay systems. Based on this criterion, in Section 5 we synthesize a sliding mode controller which asymptotically stabilizes the system for any value of the delay less than an upperbound obtained via solving a convex optimization problem. Simulation results are discussed in Section 6. Finally, Section 7 presents some conclusions.

\section{$1.1 \quad$ Notation}

The notation is fairly standard. The matrix inequality $M>0(M<0)$ means that $M$ is positive (respectively, negative) definite; $I$ denotes the identity matrix of an appropriate dimension. The vector norm $\|e\|$ stands for the Euclidean norm of a vector $e$ whereas the matrix norm

$$
\|M\|=\sup _{\|e\|=1}\|M e\|
$$

stands for the induced norm of a matrix $M ; L_{2}^{n}[-\tau, 0]$ denotes the Hilbert space of square integrable $(n)$-vector functions defined on the segment $[-\tau, 0]$. 


\section{Problem statement}

In this paper, we focus on time-delay systems that are representable by means of a nonlinear change of state coordinates and a feedback transformation, in the form

$$
\left\{\begin{array}{cc}
\frac{d z_{1}(t)}{d t}= & \sum_{i=1}^{2}\left(A_{1 i} z_{i}(t)+B_{1 i} z_{i}(t-\tau)\right) \\
& +p_{1}\left(t, z_{1}(t)\right)+p_{2}\left(t, z_{1}(t-\tau)\right) \\
\frac{d z_{2}(t)}{d t}= & \sum_{i=1}^{2}\left(A_{2 i} z_{i}(t)+B_{2 i} z_{i}(t-\tau)\right) \\
z(t)=\phi(t) & \text { for } t \in\left[-\tau+f\left(t, z_{t}\right)\right.
\end{array}\right.
$$

Hereinafter, $z(t)=\left(z_{1}, z_{2}\right)^{T}$ is the state vector with components $z_{1} \in \mathbb{R}^{n}$ $z_{2} \in \mathbb{R}^{r}, A_{i j}, B_{i j} i, j=1,2$ are constant matrices of corresponding dimensions, $u \in \mathbb{R}^{r}$ is the input vector; $p_{1}, p_{2} \in \mathbb{R}^{n}$ and $f \in \mathbb{R}^{r}$ are external disturbances, $\phi$ is the initial piecewise continuous function defined on $[-\tau, 0], z_{t}(\theta)$ is the function associated with $z$ and defined on $[-\tau, 0]$ by $z_{t}(\theta)=z(t+\theta)$. Since the delay value $\tau$ is typically unknown a priori, in the sequel we shall also use the notation $z_{t, \tau_{\max }}(\theta)$ for the function $z_{t, \tau_{\max }}(\theta)=z(t+\theta)$ defined on some larger interval $\left[-\tau_{\max }, 0\right]$ with $\tau_{\max }>\tau$.

Setting

$$
\begin{array}{r}
A_{0}=\left(\begin{array}{ll}
A_{11} & A_{12} \\
A_{21} & A_{22}
\end{array}\right), B_{0}=\left(\begin{array}{ll}
B_{11} & B_{12} \\
B_{21} & B_{22}
\end{array}\right) \\
P_{i}=\left(\begin{array}{c}
p_{i} \\
0
\end{array}\right), i=1,2, C=\left(\begin{array}{c}
0 \\
I
\end{array}\right), F=\left(\begin{array}{c}
0 \\
f
\end{array}\right)
\end{array}
$$

system (1) can be simplified to

$$
\dot{z}(t)=A_{0} z(t)+B_{0} z(t)+C u+P_{1}(t, z(t))+P_{2}(t, z(t))+F\left(t, z_{t}\right) .
$$

Although our investigation is confined to time-delay systems with delayed states, however, the extension to the case of delayed inputs is straightforward. Indeed, let a system with an input delay be governed by

$$
\left\{\begin{array}{l}
\dot{x}(t)=A x(t)+B x(t-\tau)+D u(t-\tau), t>0 \\
x(t)=\psi_{1}(t), t \in[-\tau, 0] \\
u(t)=\psi_{2}(t), t \in[-\tau, 0]
\end{array}\right.
$$

where $x \in \mathbb{R}^{n} ; A, B$ are constant $n \times n$ matrices, $D$ is a $n \times m$ matrix, $u \in \mathbb{R}^{m}$ is the input vector, $\psi_{1}$ and $\psi_{2}$ are piecewise continuous functions. Then, an additional input integrator transforms the system into the above form

$$
\left\{\begin{array}{l}
\frac{d z_{1}(t)}{d t}=A z_{1}(t)+B z_{1}(t-\tau)+D z_{2}(t-\tau) \\
\frac{d z_{2}(t)}{d t}=v
\end{array}\right.
$$


with the control input $v \in \mathbb{R}^{m}$.

Throughout the paper, the following assumptions are made for technical reasons:

A1) $p_{1}(t, z(t))$ and $p_{2}(t, z(t-\tau))$ are Lipschitz continuous and satisfy the linear growth conditions $\left\|p_{1}(t, z(t))\right\| \leq \alpha_{1}\|z(t)\|,\left\|p_{2}(t, z(t-\tau))\right\| \leq \alpha_{2}\|z(t-\tau)\|$ with some positive constants $\alpha_{1}, \alpha_{2}$.

A2) $f$ is Lipschitz continuous and it is bounded $\left\|f\left(t, z_{t}\right)\right\| \leq \Psi\left(t, z_{t, \tau_{\max }}\right)$ by a continuous functional $\Psi\left(t, z_{t, \tau_{\max }}\right)$, known a priori.

A3) $\left(A_{11}+B_{11}, A_{12}+B_{12}\right)$ is controllable.

Assumptions A1 and A2, coupled together, guarantee the the well-posedness of the unforced FDE (1) with $u=0$. Apart from this, Assumption A2 allows us to reject the uncertain disturbance $f$ by a bounded-gain feedback. Assumption A3 means the controllability of the delay-free system (1) and, in addition, it ensures that time-delay systems of the form (1) are stabilizable for all $\tau \leq \tau_{\max }$ with some positive, sufficiently small $\tau_{\max }$.

The goal of the present investigation is to construct a stabilizing sliding mode controller of (1) that makes the value of $\tau_{\max }$ as large as possible.

\section{Background materials on discontinuous time- delay systems}

Since the closed-loop system, driven by a sliding mode controller, is governed by a FDE with discontinuous right-hand side, the precise meaning of such an equation should first be defined. For this purpose, let us represent the time-delay system (3) as a dynamic system

$$
\frac{d \zeta_{t}}{d t}=\mathcal{A} \zeta_{t}+\mathcal{C} u+\mathcal{P}_{1}(t, \mu(t))+\mathcal{P}_{2}(t, \nu(-\tau))+\mathcal{F}\left(t, \nu_{t}\right)
$$

evolving in the Hilbert space $M_{2}^{n+r}=\mathbb{R}^{n+r} \times L_{2}^{n+r}[-\tau, 0]$ where the state vector $\zeta_{t}=\left(\mu(t), \nu_{t}(\cdot)\right)^{T}$ at a time moment $t$ consists of the components $\mu(t)=$ $z(t) \in \mathbb{R}^{n+r}$ and $\nu_{t}(\theta)=z_{t}(\theta) \in L_{2}^{n+r}[-\tau, 0]$, and the linear operators

$$
\mathcal{A}: \mathcal{D}(\mathcal{A}) \subset M_{2}^{n+r} \rightarrow M_{2}^{n+r}, \mathcal{C}: \mathbb{R}^{r} \rightarrow M_{2}^{n+r}
$$

and nonlinear operators

$$
\mathcal{P}_{i}: \mathbb{R}^{1+n+r} \rightarrow M_{2}^{n+r}, i=1,2, \mathcal{F}: M_{2}^{1} \rightarrow M_{2}^{n+r}
$$

are given by

$$
\begin{aligned}
& \mathcal{A}\left(\begin{array}{c}
\mu \\
\nu(\theta)
\end{array}\right)=\left(\begin{array}{c}
A_{0} \mu+B_{0} \nu(-\tau) \\
\frac{d \nu}{d \theta}
\end{array}\right), \\
& \mathcal{C}=\left(\begin{array}{c}
C u \\
0
\end{array}\right), \mathcal{P}_{i}=\left(\begin{array}{c}
P_{i} \\
0
\end{array}\right), \mathcal{F}=\left(\begin{array}{c}
F \\
0
\end{array}\right) .
\end{aligned}
$$


Apparently, $\mathcal{C}$ is a bounded operator on $\mathbb{R}^{r}$ whereas the nonlinear operators $\mathcal{P}_{i}, i=1,2$ and $\mathcal{F}$ are Lipschitz continuous on their domains due to Assumptions A1 and A2. In turn, it is well-known [5], that the infinitesimal operator $\mathcal{A}$ generates a (strongly continuous) $C_{0}$-semigroup on $M_{2}^{n+r}$ and its domain

$$
\mathcal{D}(\mathcal{A})=\left\{\zeta=(\mu, \nu)^{T} \in M_{2}^{n+r}: \frac{d \nu}{d \theta} \in L_{2}^{n+r}[-\tau, 0] \text { and } \nu(0)=\mu\right\}
$$

is dense in $M_{2}^{n+r}$.

Thus, solutions of the Hilbert space-valued dynamic system (6) with discontinuous right-hand side can rigorously be defined in the sense of [16]. For convenience of the reader, we recall the basic idea of that definition.

Let the control input $u(\zeta)$ undergo discontinuities on some linear manifold $\mathcal{G} \zeta=0$ in the state space and let $u(\zeta)$ be continuously differentiable beyond this manifold. Then trajectories of (6) are well-defined in the conventional sense whenever they are beyond the discontinuity manifold whereas in a vicinity of this manifold the original system is replaced by a related system, whose solutions also exist in the conventional sense. A motion along the discontinuity manifold, if any, is then obtained by making the characteristics of the new system approach those of the original one. Such a motion is further referred to as a sliding mode that has become standard in the literature.

It is worth noting that given an initial condition $\zeta_{0}=(\phi(0), \phi(\cdot)) \in M_{2}^{n+r}$, the Cauchy problem, thus stated for system (6) with the assumptions above, proves to be well-posed whenever the operator $\mathcal{G C}$ is continuously invertible. Just in case, the sliding motion of the system on the discontinuity manifold is governed by the so-called sliding mode equation derived through the equivalent control method.

The extension of the equivalent control method to infinite-dimensional systems has been made in $[15,16]$. According to this method, the sliding mode equation is obtained by substituting the solution

$$
u_{e q}=-[\mathcal{G C}]^{-1} \mathcal{G}\left[\mathcal{A} \zeta_{t}+\mathcal{P}_{1}(t, \mu(t))+\mathcal{P}_{2}(t, \nu(-\tau))+\mathcal{F}\left(t, \nu_{t}\right)\right]
$$

of the equation $\mathcal{G} \dot{\zeta}=0$ into (6) for $u$.

The sliding mode equation plays an important role in the subsequent stability analysis of the discontinuous time delay system in question. Since this equation contains no discontinuities in the right-hand side, its stability is established via standard techniques.

\section{Delay-dependent stability criterion}

For later use, we derive delay-dependent stability conditions for the time-delay system

$$
\begin{gathered}
\frac{d x(t)}{d t}=A x(t)+B x(t-\tau)+p_{1}(t, x(t))+p_{2}(t, x(t-\tau)) \\
x \in \mathbb{R}^{n}, x(t)=\zeta(t) \text { for } t \in[-\tau, 0]
\end{gathered}
$$


with a piece-wise continuous initial function $\zeta(t)$, some constant matrices $A, B$, and the same nonlinearities as before. Our objective is to find an upperbound $\tau_{\max }$ of admissible delay values $\tau$ such that the above system, while being asymptotically stable with $\tau=0$, is so for all delays $0 \leq \tau<\tau_{\max }$. This upperbound $\tau_{\max }$ relates to a positive solution of the following optimization problem

$$
\left(\begin{array}{ccccccc}
\multicolumn{8}{c}{\tau_{\max }=\sup \tau} \\
H(\tau) & P & P & \tau P B & \tau P B & \tau P B A & \tau P B^{2} \\
P & -\gamma_{1} I & 0 & 0 & 0 & 0 & 0 \\
P & 0 & -\gamma_{2} I & 0 & 0 & 0 & 0 \\
\tau B^{T} P & 0 & 0 & -\gamma_{3} \tau I & 0 & 0 & 0 \\
\tau B^{T} P & 0 & 0 & 0 & -\gamma_{4} \tau I & 0 & 0 \\
\tau A^{T} B^{T} P & 0 & 0 & 0 & 0 & -\tau R_{1} & 0 \\
\tau B^{2 T} P & 0 & 0 & 0 & 0 & 0 & -\tau R_{2}
\end{array}\right)<0
$$

over all positive constants $\gamma_{i}, i=1,2,3,4$ and symmetric positive definite matrices $P, R_{1}, R_{2} \in \mathbb{R}^{n \times n}$, and $H(\tau)=\left(\left(E^{T} P+P E\right)+\left(\gamma_{1} \alpha_{1}^{2}+\gamma_{2} \alpha_{2}^{2}\right) I\right)+\tau\left(\gamma_{3} \alpha_{1}^{2}+\right.$ $\left.\gamma_{4} \alpha_{2}^{2}\right) I+\tau R_{1}+\tau R_{2}$, with $E=A+B$.

Theorem 1 Let the matrix $E=A+B$ be Hurwitz. For $\alpha_{1}, \alpha_{2}>0$ sufficiently small, the above optimization problem has a positive solution $\tau_{\text {max }}$ and system (8) is globally asymptotically stable for each delay value $\tau \in\left[0, \tau_{\max }\right)$.

Proof. Consider the following Lyapunov-Krasovskii functional

$$
\begin{gathered}
V\left(z_{t}\right)=V_{1}\left(z_{t}\right)+V_{2}\left(z_{t}\right)+V_{3}\left(z_{t}\right) \\
V_{1}\left(z_{t}\right)=z^{T}(t) P z(t) \\
V_{2}\left(z_{t}\right)=\int_{t-\tau}^{t} \int_{s}^{t} z^{T}(w)\left(R_{1}+\gamma_{3} \alpha_{1}^{2} I_{n}\right) z(w) d w d s \\
+\int_{t-2 \tau}^{t-\tau} \int_{s}^{t} z^{T}(w)\left(R_{2}+\gamma_{4} \alpha_{2}^{2} I_{n}\right) z(w) d w d s \\
V_{3}\left(z_{t}\right)=\gamma_{2} \alpha_{2}^{2} \int_{t-\tau}^{t} z^{T}(w) z(w) d w d s
\end{gathered}
$$

with $P, R_{1}, R_{2}$ symmetric positive definite matrices, and positive $\gamma_{i}, i=1,2,3,4$. The functional $V\left(z_{t}\right)$ is positive definite and radially unbounded. Using $z(t-$ $\tau)=-\int_{t-\tau}^{t} \dot{z}(\theta) d \theta+z(t)$, we can rewrite the system in the form

$$
\begin{aligned}
\frac{d z(t)}{d t} & =(A+B) z(t) \\
& -B \int_{t-\tau}^{t} A z(w)+B z(w-\tau)+p_{1}(w, z(w))+p_{2}(w, z(w-\tau)) d w \\
& +p_{1}(t, z(t))+p_{2}(t, z(t-\tau))
\end{aligned}
$$


Let us now calculate the derivative of $V\left(z_{t}\right)$ along the trajectories of the system (8):

$$
\begin{aligned}
\dot{V}_{1} & =z^{T}\left(E^{T} P+P E\right) z+2 z^{T} P p_{1}+2 z^{T} P p_{2} \\
& -2 z^{T} P B A \int_{t-\tau}^{t} z(w) d w \\
& -2 z^{T} P B^{2} \int_{t-\tau}^{t} z(w-\tau) d w \\
& -2 z^{T} P B \int_{t-\tau}^{t} p_{1}(w, z(w)) d w \\
& -2 z^{T} P B \int_{t-\tau}^{t} p_{2}(w, z(w-\tau)) d w, \\
\dot{V}_{2} & =\tau z^{T}\left(R_{1}+\gamma_{3} \alpha_{1}^{2} I\right) z+\tau z^{T}\left(R_{2}+\gamma_{4} \alpha_{2}^{2} I\right) z \\
& -\int_{t-\tau}^{t} z^{T}(w)\left(R_{1}+\gamma_{3} \alpha_{1}^{2} I\right) z(w) d w \\
& -\int_{t-\tau}^{t} z^{T}(w-\tau)\left(R_{2}+\gamma_{4} \alpha_{2}^{2} I\right) z(w-\tau) d w, \\
\dot{V}_{3} & =\gamma_{2} \alpha_{2}^{2} z^{T}(t) z(t)-\gamma_{2} \alpha_{2}^{2} z^{T}(t-\tau) z(t-\tau) .
\end{aligned}
$$

Due to the well-known inequality $2 u^{T} v \leq \alpha u^{T} R u+\alpha^{-1} v^{T} R^{-1} v$, valid for any vectors $u, v \in \mathbb{R}^{n}$, symmetric positive definite matrix $R \in \mathbb{R}^{n \times n}$ and positive constant $\alpha$, the following six inequalities are in force:

$$
\begin{aligned}
& 2 z^{T} P p_{1} \leq z^{T}\left(\gamma_{1}^{-1} P^{2}+\gamma_{1} \alpha_{1}^{2} I\right) z, \\
& 2 z^{T} P p_{2} \leq \gamma_{2}^{-1} z^{T} P^{2} z+\gamma_{2} \alpha_{2}^{2} z(t-\tau)^{T} z(t-\tau), \\
& -2 z^{T} P B A \int_{t-\tau}^{t} z(w) d w \leq \tau z^{T} Z_{1} z+\int_{t-\tau}^{t} z^{T}(w) R_{1} z(w) d w \\
& -2 z^{T} P B^{2} \int_{t-\tau}^{t} z(w-\tau) d w \leq \tau z^{T} Z_{2} z+\int_{t-\tau}^{t} z^{T}(w-\tau) R_{2} z(w-\tau) d w, \\
& -2 z^{T} P B \int_{t-\tau}^{t} p_{1}(w, z(w)) d w \leq \tau \gamma_{3}^{-1} z^{T} Z_{3} z+\gamma_{3} \alpha_{1}^{2} \int_{t-\tau}^{t} z^{T}(w) z(w) d w, \\
& -2 z^{T} P B \int_{t-\tau}^{t} p_{2}(w, z(w-h)) d w \leq \tau \gamma_{4}^{-1} z^{T} Z_{3} z+\gamma_{4} \alpha_{2}^{2} \int_{t-\tau}^{t} z^{T}(w-\tau) z(w-\tau) d w
\end{aligned}
$$

with $I$ identity matrix, $Z_{1}=P B A R_{1}^{-1} A^{T} B^{T} P, Z_{2}=P B^{2} R_{2}^{-1} B^{2 T} P, Z_{3}=$ $P B B^{T} P, \gamma_{i}>0, i=1,2,3,4$. Now it follows from (11) that the inequality

$$
\dot{V}\left(z_{t}\right) \leq z^{T} M(\tau) z
$$

holds for

$$
\begin{aligned}
& M(\tau)=\left(E^{T} P+P E\right)+\left(\gamma_{1}^{-1}+\gamma_{2}^{-1}\right) P^{2}+ \\
& \left(\gamma_{1} \alpha_{1}^{2}+\gamma_{2} \alpha_{2}^{2}+\tau \gamma_{3} \alpha_{1}^{2}+\tau \gamma_{4} \alpha_{2}^{2}\right) I+\tau\left(R_{1}+R_{2}\right) \\
& +\tau P B A R_{1}^{-1} A^{T} B^{T} P+\tau P B^{2} R_{2}^{-1} B^{2 T} P+\tau \gamma_{3}^{-1} P B B^{T} P+\tau \gamma_{4}^{-1} P B B^{T} P
\end{aligned}
$$


The matrix $E$ is Hurwitz and hence $M(0)=\left(E^{T} P+P E\right)+\left(\gamma_{1}^{-1}+\gamma_{2}^{-1}\right) P^{2}+$ $\left(\gamma_{1} \alpha_{1}^{2}+\gamma_{2} \alpha_{2}^{2}\right) I$ is negative definite if $P$ is a symmetric positive definite solution $P$ of the Lyapunov equation $E^{T} P+P E=-I$ and positive constants $\gamma_{1}, \gamma_{2}$, are sufficiently small. By continuity $M(\tau)$ remains negative definite for sufficiently small positive $\tau$. Since by Schur's lemma [1], $M(\tau)<0$ if and only if inequality (9) holds, it follows that the afore-given optimization problem has a positive solution $\tau_{\max }$. Thus, the matrix $M(\tau)$ is negative definite for all $\tau \in\left[0, \tau_{\max }\right)$ and system (8) is therefore globally asymptotically stable for all delays $0 \leq \tau$ $<\tau_{\max }$. Theorem 1 is proven.

Remark 2 The inequality (9) is a linear matrix inequality in $P, R_{1}, R_{2}, \gamma_{1}, \gamma_{2}, \gamma_{3}, \gamma_{4}$ and can efficiently be solved by convex optimization algorithms (see [1]).

Example 3 (Comparison with other criteria)

Let system (8) be specified by

$$
A=\left(\begin{array}{cc}
-2 & 0 \\
0 & -1
\end{array}\right), B=\left(\begin{array}{cc}
-1 & 0 \\
-1 & -1
\end{array}\right)
$$

and let $p_{1}$ and $p_{2}$ be such that $\left\|p_{1}\right\| \leq 0.2\left\|z_{1}\right\|$ and $\left\|p_{2}\right\| \leq 0.2\left\|z_{1}(t-\tau)\right\|$. Using Theorem 1, we find $\tau_{\max }=0.623$ whereas the largest upperbound of time-delay available in the literature ( see $[14,19,20])$ is $\tau_{\max }=0.4428$. It should also be noted that the delay independent criterion [24] cannot provide any conclusion. Thus, the example shows that the proposed criterion makes a step beyond the existing results.

\section{$5 \quad$ Sliding mode state feedback controller}

The following state feedback control law

$$
u=-\left[\Omega_{0}\left(t, z_{t, \tau_{\max }}\right)+\Psi\left(t, z_{t}\right)+g\|s\|^{\varepsilon}\right] \frac{s(z(t))}{\|s(z(t))\|}, g>0, \varepsilon \in[0,1)
$$

is proposed to drive the uncertain time-delay system (1) to a linear manifold $s(z)=0$ with

$$
s(z)=z_{2}+K z_{1}
$$

in finite time thereby globally asymptotically stabilizing the system for each $\tau \in\left[0, \tau_{\max }\right)$. Hereinafter,

$$
\Omega\left(t, z_{t}\right)=\sum_{i=1}^{2}\left[A_{2 i} z_{i}(t)+B_{2 i} z_{i}(t-\tau)+K\left(A_{1 i} z_{i}(t)+B_{1 i} z_{i}(t-\tau)\right]\right.
$$

is a linear functional on the trajectories of the controlled system (1), and

$$
\Omega_{0}\left(t, z_{t, \tau_{\max }}\right)=\sup _{\theta \in\left[-\tau_{\max }, 0\right]}\left(\| \Omega\left(t, z_{t}(\theta) \|\right.\right.
$$


is its upperbound on a time interval $\theta \in\left[-\tau_{\max }, 0\right]$ whereas $\tau_{\max }>0$ and $K \in \mathbb{R}^{n \times n}$ are subject to the optimization

$$
\begin{aligned}
& \tau_{\max }=\sup \tau
\end{aligned}
$$

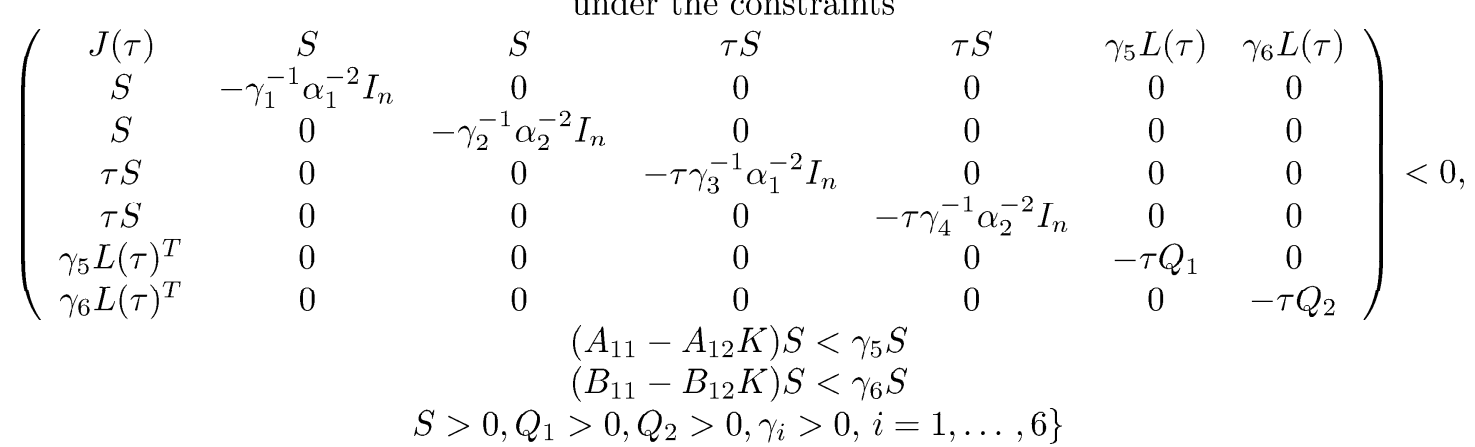

with symmetric positive definite matrices $S, Q_{1}, Q_{2} \in \mathbb{R}^{(n-m) \times(n-m)}$,

$$
\begin{array}{r}
J(\tau)=\left[\left(A_{11}+B_{11}\right)-\left(A_{12}+B_{12}\right) K\right] S+S\left[\left(A_{11}+B_{11}\right)-\left(A_{12}+B_{12}\right) K\right]^{T} \\
+\left[\gamma_{1}^{-1}+\gamma_{2}^{-1}+\tau\left(\gamma_{3}^{-1}+\gamma_{4}^{-1}\right) \gamma_{6}^{2}\right] I+\tau\left(Q_{1}+Q_{2}\right),
\end{array}
$$

and $L(\tau)=\tau\left(B_{11}-B_{12} K\right) S$.

It is of interest to note that if system (1) is only affected by external disturbances $f\left(t, z_{t}\right)$, vanishing in the origin, then an upperbound $\Psi\left(t, z_{t, \tau_{\max }}\right)$, such that $\Psi(t, 0)=0$ for all $t$, becomes available so that the corresponding controller (13) with a positive parameter $\varepsilon$ is continuous in the origin with no undesirable effects of switching in the steady state.

It should be pointed out that the control law (13) appears to be applicable even if the system delay is unknown a priori.

We are now in a position to state the main result of this section.

Theorem 4 For upperbounds $\alpha_{1}, \alpha_{2}$ sufficiently small the optimization problem (15) has a positive solution $\tau_{\max }$ and the closed-loop system (1), (13) is globally asymptotically stable for each delay value $\tau \in\left[0, \tau_{\max }\right)$.

Proof. We break up the proof in 3 simple steps. Firstly, we prove that the discontinuity manifold $s(z)=0$ is reached in finite time, secondly, we derive the state equations along the manifold $s(z)=0$, and thirdly, we prove the asymptotic stability of the system motion on this manifold, thereby completing the proof of Theorem 4 .

1) Attractiveness of the manifold. Taking into account that the time derivative of $s(z(t))$ along the solutions of (1) is given by

$$
\dot{s}(z(t))=\Omega\left(t, z_{t}\right)+u+f\left(t, z_{t}\right)
$$


differentiating of the Lyapunov-Krasovskii functional

$$
V(t)=\frac{s^{T}(z(t)) s(z(t))}{2} .
$$

on the trajectories of the closed-loop system under Assumptions 1-3 yields

$$
\begin{aligned}
\dot{V}(t) & =s^{T}(z(t)) \dot{s}(z(t)) \\
& =s^{T}(z(t))\left\{\Omega\left(t, z_{t}\right)+f\left(t, z_{t}\right)-\left[\Omega_{0}\left(t, z_{t, \tau_{\text {max }}}\right)+\Psi\left(t, z_{t, \tau_{\text {max }}}\right) g\|s\|^{\varepsilon}\right] \frac{s}{\|s\|}\right\} \\
& \leq-g \| s\left(z(t) \|^{\varepsilon+1} \leq-g V(t)^{\frac{\varepsilon+1}{2}} .\right.
\end{aligned}
$$

Since an arbitrary solution of the latter inequality is well-known to vanish after a finite time moment, the finite-time convergence of the trajectories of the closed-loop system to the discontinuity manifold $s(z)=0$ is concluded. In order to reproduce this conclusion, one should note that for all $t \geq 0$ arbitrary solution $V(t)$ to the latter inequality is dominated $V(t) \leq V_{0}(t)$ by the solution to the differential equation $\dot{V}_{0}(t)=-g V(t)^{\frac{\varepsilon+1}{2}}$, initialized with the same initial condition $V_{0}(0)=V(0)$. Since $V_{0}(0)=0$ for all $t \geq \frac{2}{g(1-\varepsilon)} V(t)^{\frac{1-\varepsilon}{2}}, V(t)$ vanishes after the finite time moment $T=\frac{2}{g(1-\varepsilon)} V(t)^{\frac{1-\varepsilon}{2}}$.

2) Sliding mode equation. In order to describe the system motion on the discontinuity manifold $s(z)=0$ one should follow the extension [15] of the equivalent control method [21] to infinite-dimensional systems driven in a Hilbert space. According to this method, the continuous solution $u_{e q}=\left[\Omega\left(t, z_{t}\right)+\right.$ $f\left(t, z_{t}\right)$ ] of the equation $\dot{s}=0$ is substituted into (1) for $u$. Thus, on the discontinuity manifold, the system is governed by the differential equation

$$
\begin{aligned}
\frac{d z_{1}(t)}{d t}= & \left(A_{11}-A_{12} K\right) z_{1}(t)+\left(B_{11}-B_{12} K\right) z_{1}(t-\tau) \\
& +p_{1}\left(t, z_{1}(t)\right)+p_{2}\left(t, z_{1}(t-\tau)\right) .
\end{aligned}
$$

3) Asymptotic stability of the reduced system. Since system (1) is controllable by assumption, the matrix $E=\left(A_{11}-A_{12} K\right)+\left(B_{11}-B_{12} K\right)$ is Hurwitz by a proper choice of the matrix $K$. Then applying Theorem 1 to system (18), we obtain that the optimization problem (15) has a positive solution $\tau_{\max }$ and the sliding mode system (18) is asymptotically stable if and only if

$$
\begin{gathered}
\left(S E^{T}+E S\right)+\left(\gamma_{1}^{-1}+\gamma_{2}^{-1}\right) I_{n-r}+\left(\gamma_{1} \alpha_{1}^{2}+\gamma_{2} \alpha_{2}^{2}+\tau \gamma_{3} \alpha_{1}^{2}+\tau \gamma_{4} \alpha_{2}^{2}\right) S^{2} \\
+\tau\left(S R_{1} S+S R_{2} S\right)+\tau B A R_{1}^{-1} A^{T} B^{T}+\tau B^{2} R_{2}^{-1} B^{2 T} \\
+\left(\tau \gamma_{3}^{-1}+\tau \gamma_{4}^{-1}\right) B B^{T}<0
\end{gathered}
$$

Under constraints

$$
Q_{1}=S R_{1} S, Q_{2}=S R_{2} S, A_{11} S-A_{12} K S<\gamma_{5} S, B_{11} S-B_{12} K S<\gamma_{6} S
$$


with some positive $\gamma_{5}, \gamma_{6}$, inequality $M(\tau)<0$ implies that

$$
\begin{aligned}
& \left.\left(S E^{T}+E S\right)+\left(\gamma_{1}^{-1}+\gamma_{2}^{-1}\right) I_{n-m}+\tau\left(\gamma_{3}^{-1}+\gamma_{4}^{-1}\right) \gamma_{6}^{2}\right) I_{n-m}+\tau\left(Q_{1}+Q_{2}\right) \\
& +\left(\gamma_{1} \alpha_{1}^{2}+\gamma_{2} \alpha_{2}^{2}+\tau \gamma_{3} \alpha_{1}^{2}+\tau \gamma_{4} \alpha_{2}^{2}\right) S^{2} \\
& +\gamma_{5}^{2} \tau\left(B_{11} S-B_{12} W\right) Q_{1}^{-1}\left(B_{11} S-B_{12} W\right)^{T} \\
& +\gamma_{6}^{2} \tau\left(B_{11} S-B_{12} W\right) Q_{2}^{-1}\left(B_{11} S-B_{12} W\right)^{T}<0 .
\end{aligned}
$$

By Schur's lemma [1], (21) is equivalent to (15), thereby concluding the global asymptotic stability of the sliding mode equation (18) for each delay value $\tau \in$ $\left[0, \tau_{\max }\right)$. Theorem 4 is thus completely proven.

Remark 5 While $\gamma_{i}>0, i=1, \ldots, 6$ being fixed, (15) is a generalized eigenvalue problem which can efficiently be solved by convex optimization. A suboptimal upperbound $\tau_{\max }$ is thus found by relaxation type algorithms.

\section{Example}

Consider system (3) with

$$
\begin{aligned}
A_{0} & =\left(\begin{array}{ccc}
2 & 0 & 1 \\
1.4 & 0.25 & 0.8 \\
-1 & 0 & 1
\end{array}\right), B_{0}=\left(\begin{array}{ccc}
-1 & 0 & 0 \\
-0.1 & 0.25 & 0.2 \\
-0.2 & 4 & 5
\end{array}\right), C=\left(\begin{array}{l}
0 \\
0 \\
1
\end{array}\right) \\
& =\left(\begin{array}{c}
0 \\
0 \\
\sin \left(z_{1}(t-\tau)\right)
\end{array}\right), P_{1}=P_{2}=0
\end{aligned}
$$

and the initial condition

$$
z(t)=\left[\begin{array}{c}
-1 \\
-0.5 \\
3
\end{array}\right] \text { for } t \in[-\tau, 0] .
$$

Let the sliding surface (14) be specified with $K=(1.317,3.979)$. Then by using semidefinite programming, system (3), (22), driven by the sliding mode controller (13) subject to $\varepsilon=0, \Psi\left(t, z_{t, \tau_{\max }}\right)=\max _{\theta \in\left[t, t-\tau_{\text {max }}\right]}\|z(t)\|, g=1.1$, is globally asymptotically stable for each delay value $\tau \in\left[0, \tau_{\max }\right]$ where $\tau_{\max }=$ 0.63. The simulation results depicted in Figure 1 are obtained with a $5^{\text {th }}$ order integration scheme of step $10^{-3}$.

By setting $\varepsilon=\frac{1}{2}$, the control input becomes continuous in the origin and the chattering phenomenon is thus drastically reduced. As shown in Figure 2, the amplitudes of the input oscillations, coming in this simulation run, are reduced from 2 to 0.02 without apparent changes in the convergence rate.

Remark 6 Comparison with previous results:

The sliding mode controllers proposed in [3, 18] cannot stabilize system (22) 
since the pair $\left(A_{0}, C\right)$ is not controllable in this case. With our approach, $\left(A_{0}+B_{0}, C\right)$-controllability relaxes this requirement. The standard state feedback, developed in [14, 24], can only attenuate vanishing disturbances. Controllers from [4], which are based on linear models over rings and reject nonvanishing disturbances, require the delay value to be known a priori.

\section{Conclusion}

Discontinuous time-delay control systems are under study. The system behavior on the manifold where the controller undergoes discontinuities is shown to be governed by the equation obtained through the equivalent control method extended to Hilbert space-valued dynamic systems. Based on this observation, sliding mode control synthesis, robust against sufficiently small delay variations and external disturbances, is developed for a class of uncertain nonlinear timedelay systems. The synthesis consists of two steps. First, a manifold, such that if confined to this manifold the time delay system has desired dynamic properties, is designed. Second, a discontinuous control law, which drives the system to this manifold in finite time, is synthesized. An admissible upperbound such that the corresponding closed-loop system remains globally asymptotically stable for all delay values less than this upperbound is derived. Theoretical results are supported by a numerical example.

\section{References}

[1] S. Boyd, L. El Ghaoui, E. Feron, and V. Balakrishan, Linear Matrix Inequalities in System and Control Theory. SIAM: Philadelphia, 1994.

[2] C.I. Byrnes, I.G. Lauko, D.S. Gilliam, and V.I. Shubov, "Output regulation for linear distributed parameter systems," IEEE Trans. Autom. Contr., vol. 45, pp. 2236-2252, 2000.

[3] H.H. Choi, " An LMI approach to sliding mode control design for a class of uncertain time-delay systems," Proc. ECC'99 European Control Conference, Karlsruhe, Germany, 1999.

[4] G. Conte and A.-M. Perdon, "Systems over rings: theory and applications," Plenary lecture, Proc. IFAC Workshop LTDS'98, Linear Time Delay Systems, Grenoble, France, p. 223-234, 1998.

[5] R.F. Curtain and H.J. Zwart, An Introduction to Infinite-Dimensional Linear Systems Theory. Springer-Verlag: New York, 1995.

[6] R. El-Khazali, "Variable structure robust control of uncertain time-delay systems," Automatica vol. 34, no. 3, pp. 327-332, 1998. 
[7] C. Foias, H. Özbay, and A. Tannenbaum, Robust Control of Infinite Dimensional Systems: Frequency Domain Methods. Springer-Verlag: London, 1996.

[8] F. Gouaisbaut, Y. Blanco, and J.P. Richard, "Robust sliding mode control of nonlinear systems with delay : a design via polytopic formulation," nternat. J. Control, 2002 (to be published).

[9] F. Gouaisbaut, M. Dambrine, and J.P. Richard, "Robust control of delay systems: a sliding mode control design via LMI," Systems 83 Control Letters, vol. 46, no. 4, pp. 219-230, 2002.

[10] B. van Keulen, $H_{\infty}$-Control for Distributed Parameter Systems: A StateSpace Approach. Boston: Birkhauser, 1993.

[11] S.G. Krein, Linear Differential Equations in Banach Space. American mathematical society: Providence, 1971.

[12] L. Levaggi, "Sliding modes in banach spaces," Differential Integral Equations, vol. 15, pp. 167-189, 2002.

[13] L. Levaggi, "Infinite dimensional systems sliding motions," European Journal of Control, 2002 (to be published).

[14] X. Li, and C.E. De Souza, "Delay -dependent robust stability and stabilization of uncertain linear delay system : a linear matrix inequality approach," IEEE Trans. Aut. Control, vol. 42, No 8, pp.1144-1148, 1997.

[15] Y. Orlov, "Discontinuous unit feedback control of uncertain infinitedimensional systems," IEEE Trans. Autom. Contr., vol. 45, pp. 834-843, 2000.

[16] Y. Orlov, and D. Dochain, "Discontinuous feedback stabilization of minimum-phase semilinear infinite-dimensional systems with application to chemical tubular reactor," IEEE Trans. Autom. Contr., vol. 47, pp. 1293-1304, 2002.

[17] Y. Orlov and V.I. Utkin, "Sliding mode control in infinite-dimensional systems," Automatica, vol.23, pp.753-757, 1987.

[18] K.K. Shyu and J.J. Yan "Robust stability of uncertain time-delay systems and its stabilization by variable structure control," Internat. J. Control, vol. 57, no. 1, pp. 237-246, 1993.

[19] J.H. Su, "Further results on the robust stability of delay dependence for linear uncertain systems," Systems \& Control letters, vol. 23, pp.375-379, 1994.

[20] J.H. Su and C.G. Huang,(1992) "Robust stability on delay dependence for linear uncertain systems", IEEE Trans. Aut. Contr., vol. 37, pp.1656-1659, 1992. 
[21] V.I. Utkin, Sliding Modes in Control Optimization. Springer - Verlag: Berlin, 1992.

[22] V.I. Utkin, J. Guldner, and J. Shi, Sliding modes in electromechanical systems London, Taylor and Francis, 1999.

[23] F. Zheng and P. M. Frank, "Finite dimensional variable structure control design for distributed delay systems," Internat. J. Control, vol. 74, no. 4, pp. 398-408, 2001.

[24] L. Xie and C.E. De Souza C.E, "Robust stabilization and disturbance attenuation for uncertain delay systems", Proc. European Control Conference, Groningen, The Netherlands, 1993.

[25] T. Zolezzi, "Variable structure control of semilinear evolution equations," Partial differential equations and the calculus of variations, Essays in honor of Ennio De Giorgi, vol.2, pp.997-1018. Birkhauser: Boston, 1989. 

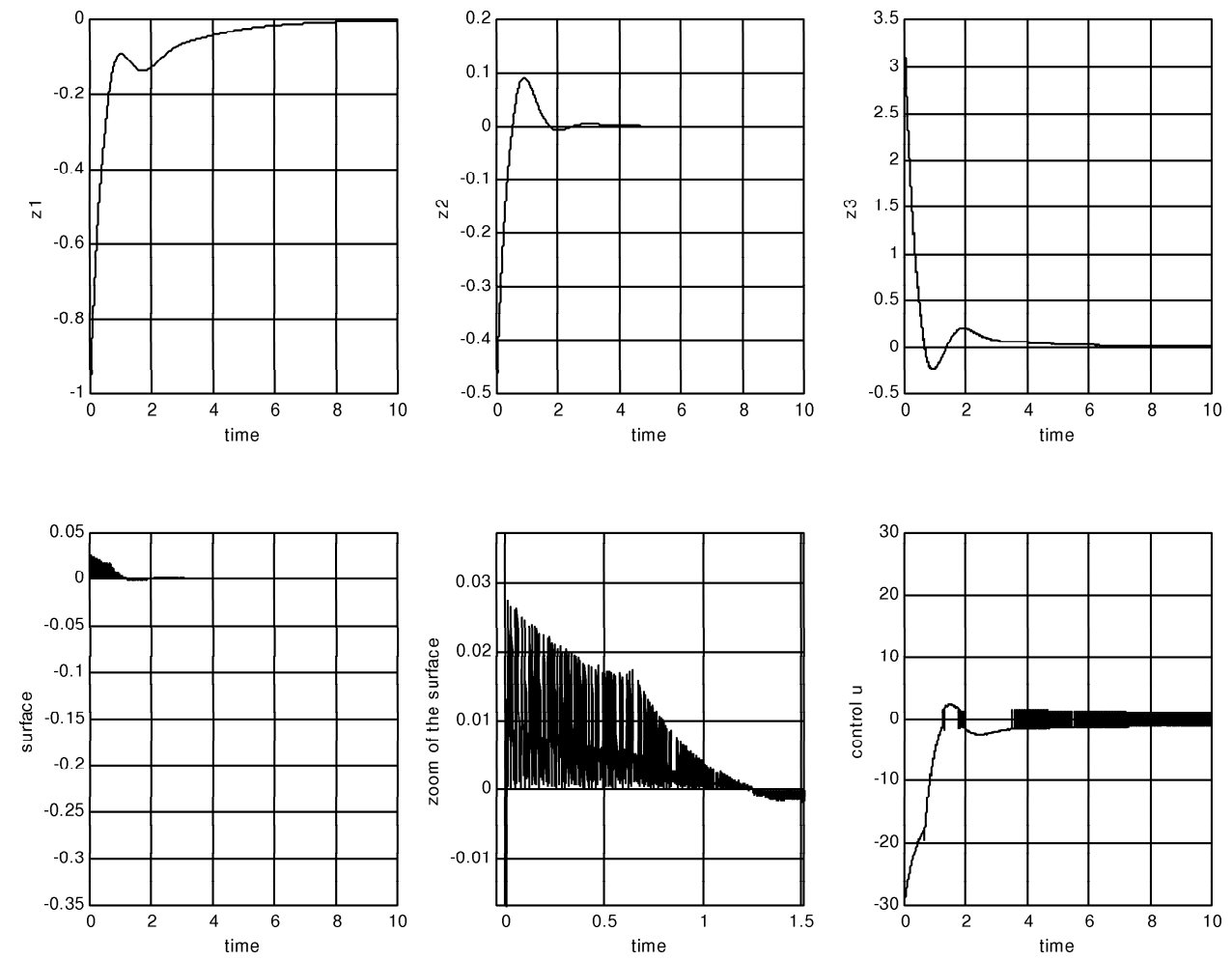

Figure 1: Response of the closed-loop system (22), (13) with $\tau=0.63$ and $\varepsilon=0$. 

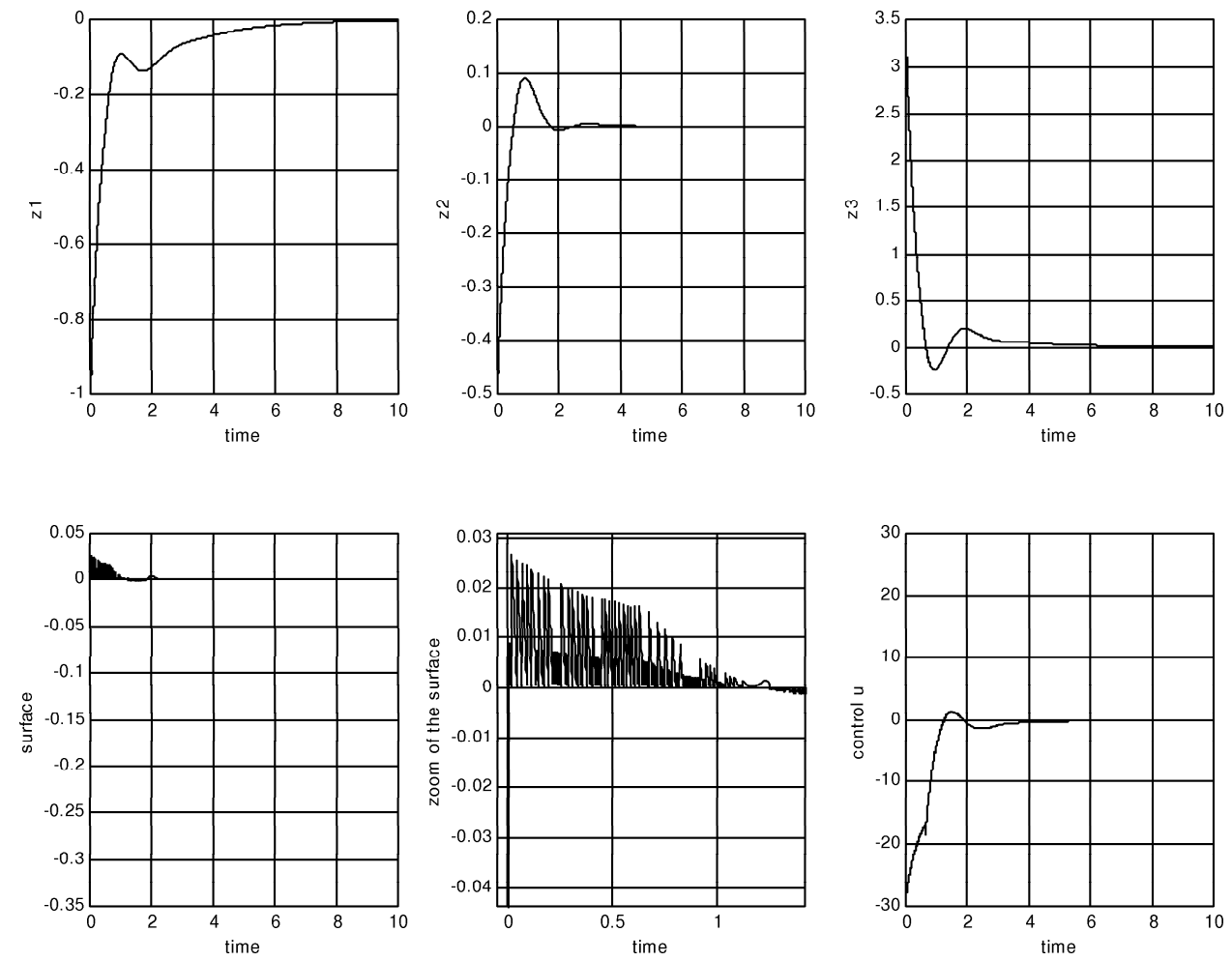

Figure 2: Response of the closed-loop system (22), (13) with $\tau=0.63$ and $\varepsilon=\frac{1}{2}$. 